\title{
Comparando el índice h con otros indicadores bibliométricos en el tema "Estudios Métricos" en el SciELO, en Ciencia de la Información
}

Comparando o índice $h$ com outros indicadores bibliométricos

na temática estudos métricos no SciELO, em Ciência da Informação

Comparing the $h$ index with other bibliometric indicators:

the case of Metric Studies in the Information Science area of SciELO

Maria Cláudia Cabrini Grácıo (1), Ely Francina Tannuri de OliveIRA (2)

(1) Faculdade de Filosofia e Ciências, Unesp, Av. Hygino Muzzi Filho, 737 Marília - São Paulo - Brasil - CEP: 17525-900, cabrini@marilia.unesp.br (2) etannuri@flash.tv.br

\begin{abstract}
Resumen
Esta investigación presenta el concepto de índice $\mathrm{h}$ y lo compara con otros indicadores bibliométricos de los investigadores más citados en los periódicos del área de Ciencia de la Información del SciELO, en la temática "Estudios Métricos". Como procedimiento de investigación, para cada uno de los 35 investigadores en análisis, han sido buscados en la base de datos Web of Science, los indicadores: índice $\mathrm{h}$, total de artículos publicados, número total de citaciones, media de citaciones y citaciones del artículo más citado. Se presentan los datos y calculada de Pearson entre estos indicadores. Los resultados mostraron mayor correlación entre el índice $\mathrm{h}$ y el total de citaciones y entre el índice $\mathrm{h}$ y el número de citaciones del artículo más citado. Se considera que el índice $\mathrm{h}$ es un indicador relevante de la producción acumulada del investigador. Sin embargo, con objeto de obtener un perfil pleno del mismo, debe ser complementado con otros indicadores.
\end{abstract}

Palabras clave: Índice h. Indicadores bibliométricos. Frente de investigación en "Estudios Métricos".Indicadores de citación.

\section{Introdução}

A crescente explosão da produção científica nas diferentes áreas do conhecimento impôs a necessidade da avaliação da ciência como um todo, bem como dos pesquisadores, grupos, instituições e países, com a finalidade de se conhecer aqueles que se destacam e que mais contribuem para o avanço do saber e subsidiar políticas científicas governamentais e de instituições.

Diversas modalidades de avaliação têm sido sugeridas. Entre elas, as análises bibliométricas têm se mostrado ferramentas confiáveis e objetivas, apesar das controvérsias e ressalvas apontadas por estudiosos da área específica de bibliometria e por pesquisadores que estão sujeitos a este processo de avaliação.

\begin{abstract}
This paper introduces the concept of h-index and compares it with other bibliometric indicators of the most cited researchers in the periodicals from the area of Information Science in SciELO, in the theme of "Metric Studies". For each one of the 35 researchers under review. Were sought for in the Web of Science database, these indicators: h-index, total of published articles, total number of citations, average of citations and quotations from the most cited article. The data and the Pearson's correlation between these indicators is presented. The results showed the highest correlation between the $\mathrm{h}$-index and the total number of citations and between the $\mathrm{h}$-index and the number of quotations from the most cited article. It is considered that the $h$ index is a relevant indicator of the accumulated production obtained by the researcher. However in order to get a full profile of each one, it must be complemented with other indicators.
\end{abstract}

Keywords: H-index. Bibliometric indicators. Research front in "Metric Studies". Citation indicators.

Assim, média de citações, total de citações, total de produções e fator de impacto são indicadores clássicos de produtividade, de relevância e de impacto de autores, periódicos, instituições, grupos ou países, nas diferentes áreas do conhecimento.

Recentemente, em 2005, um novo indicador bibliométrico - 0 índice $h-$ foi proposto pelo físico Jorge E. Hirsch, da Universidade da Califórnia, inicialmente com a finalidade de avaliar a produção científica individual de cada pesquisador, visando estimar a importância e o impacto da sua contribuição acumulada de pesquisa.

Nesta pesquisa, focou-se o conjunto de pesquisadores mais citados na temática "Estudos Métricos" nos periódicos da área de Ciência da 
Informação disponibilizados no SciELO, biblioteca eletrônica considerada relevante pelas publicações de periódicos nucleares e consolidados nas diferentes áreas do conhecimento. Esses pesquisadores foram levantados em pesquisa anteriormente realizada sobre análise de citação e cocitação (Grácio, Oliveira e Matos, 2009).

A proposta é analisar o impacto e a visibilidade dos pesquisadores na temática "Estudos Métricos", tomando-se como referência os indicadores disponibilizados na base Web of Science, comparando o índice h com os demais indicadores.

Justifica-se esta pesquisa pela contribuição oferecida para a compreensão dos indicadores como descritores da trajetória acadêmica dos estudiosos mais citados. Acrescente-se ainda a carência de estudos relativos a esses indicadores, especialmente o índice h, na temática "Estudos Métricos", subárea relevante na Ciência da Informação.

\section{Objetivos}

Objetiva-se analisar o índice $\mathrm{h}$ e outros indicadores de impacto e relevância dos pesquisadores mais citados na temática "Estudos Métricos", nos periódicos da área de Ciência da Informação do SciELO, a fim de descrever seu desempenho e cotejar, entre si, os indicadores.

De forma mais especifica, busca-se obter o índice $\mathrm{h}$, a média de citações, o número total de citações, número de citações do artigo mais citado e o total de artigos publicados por autor, presentes na Web of Science, e observar a correlação entre tais indicadores. Pretende-se realizar uma análise comparativa dos indicadores como descritores de medidas de produtividade e visibilidade científica dos pesquisadores mais citados no tema "Estudos Métricos" dos periódicos do SciELO, na área de Ciência da Informação.

\section{Apresentando os descritores de produtividade e visibilidade científica}

A Scientific Electronic Library Online - SciELO é uma biblioteca eletrônica que abrange uma coleção selecionada de periódicos científicos brasileiros e resultou de um projeto de pesquisa da Fundação de Amparo à Pesquisa do Estado de São Paulo (FAPESP), em parceria com o Centro Latino-Americano e do Caribe de Informação em Ciências da Saúde (BIREME).

Seu objetivo é o desenvolvimento de uma metodologia comum para a preparação, armazenamento, disseminação e avaliação da produção científica em formato eletrônico.
Segundo (Meneghini, 1998, p. 220), visa

tornar mais visível a literatura científica brasileira e, ao mesmo tempo, criar uma base de dados que possa ser utilizada para a obtenção de informações úteis em termos de sociologia da ciência no Brasil, que permita, entre outras coisas, o estabelecimento de estratégias e de políticas de gestão científica.

Atualmente, são publicados 204 periódicos correntes, envolvendo 8 campos de conhecimentos, tais como, Ciências Agrárias, Ciências Biológicas, Ciências da Saúde, Ciências Exatas e da Terra, Ciências Sociais e Aplicadas, Engenharias, Lingüística, Letras e Artes. No campo de Ciências Sociais Aplicadas, se encontra a área Ciência da Informação, com publicação de dois periódicos: Perspectivas em Ciência da Informação e Ciência da Informação.

O primeiro, Perspectivas em Ciência da Informação, é uma publicação quadrimestral da Escola de Ciência da Informação da UFMG (Universidade Federal de Minas Gerais).

O segundo, Ciência da Informação, trata de publicações quadrimestrais de trabalhos inéditos na área ou que apresentam resultados de estudos e investigações sobre as atividades e estudos de investigação do setor de informação em Ciência e Tecnologia (C\&T). O periódico em questão tem forte atuação no campo da Ciência da Informação e no setor de Informação em $C \& T$, por veicular contribuições significativas de experiências nacionais e internacionais endereçadas a um público constituído especialmente pela comunidade acadêmica de profissionais e investigadores, não só da Ciência da Informação, mas também de áreas com temáticas afins.

Além de pertencerem a uma biblioteca digital reconhecida internacionalmente, os dois periódicos são os que apresentam melhor avaliação em relação aos demais periódicos brasileiros da área, pela Coordenação de Aperfeiçoamento de Pessoal de Nível Superior (CAPES), que afere a qualidade dos artigos, a partir da análise da qualidade dos veículos de divulgação, ou seja, periódicos científicos.

Compreende-se aqui a expressão "Estudos Métricos" como o conjunto de estudos relacionados à avaliação da ciência produzida, nos diferentes suportes, baseados em recursos quantitativos como ferramentas de análise. Fundamentados em conceitos da sociologia da ciência e em conceitos matemáticos, estatísticos e computacionais, são estudos de natureza teórico-conceitual, quando contribuem para o avanço do conhecimento da própria temática, propondo novos conceitos e indicadores para a área, bem como reflexões e análises relativas à 
área. São também de natureza metodológica, quando se propõem a dar sustentação aos trabalhos de natureza teórica da área onde estão aplicados.

Os Estudos Métricos desenvolveram-se a partir da bibliometria, cientometria, webometria, tendo como tema "maior" a informetria. Mais modernamente, surge também a patentometria. Os Estados Unidos, Bélgica, Holanda e Espanha, entre outros, são os países precursores destes estudos.

Nesta pesquisa, os próprios indicadores bibliométricos, especificamente os de produção e de citação, se configuram como procedimentos metodológicos, a fim de analisar a frente de pesquisa desta temática, a partir do ranking dos pesquisadores mais citados, conforme visto em estudo anterior.

O indicador básico de produção é constituído pela contagem do número de publicações do pesquisador, que busca, através da sua produtividade, refletir seu impacto junto à comunidade científica a qual pertence. No entanto, estudiosos têm apontado a independência entre produtividade e impacto de pesquisador, considerando que nem sempre alto volume de produção acadêmica significa visibilidade e reconhecimento pelos pares.

Entre os indicadores de citação clássicos para a avaliação individual do pesquisador, têm-se o número total de citações de cada pesquisador e a média de citações por trabalho publicado, que buscam refletir o impacto, a influência e a sua visibilidade junto à comunidade científica. No entanto, estudiosos da área apontam questões associadas ao uso destes indicadores, tomados em sua forma bruta na análise da produção científica. Essas questões se relacionam às diferentes naturezas das áreas onde se aplicam, às motivações para citação, à própria natureza da publicação - se de revisão ou original -, à barreira linguística e à origem da publicação - se de países periféricos ou mainstream.

Em decorrência das questões apontadas, estudiosos buscam continuamente novos indicadores a fim de sanar as lacunas.

Jorge Hirsch, em 2005, propôs o índice h, um indicador de número único, como um modo simples e útil de caracterizar a produção científica de um pesquisador. Trata-se de um índice que propicia uma estimativa da importância, da relevância, do reconhecimento e da contribuição da pesquisa acumulada, para a área.

$\mathrm{O}$ autor define o índice $\mathrm{h}$ como "a scientist has index $h$ if $h$ of his /her $N_{p}$ papers have at least $h$ citations each, and the other $\left(N_{p}-h\right)$ papers have no more than h citation each" (Hirsch, 2005, p. 1), onde $\mathrm{N}_{\mathrm{p}}$ é o número total de artigos publicados.

Para se obter o valor do índice $\mathrm{h}$ é necessário construir uma lista, atribuindo um número de ordem aos artigos publicados pelo autor, em função da ordem decrescente do número de citações recebidas. Dessa forma, constroem-se duas listas de números: uma ascendente, referente ao número de ordem do artigo publicado, e outra decrescente, segundo o número de citações do artigo. O índice $\mathrm{h}$ será o valor para o qual o número de citações do artigo é menor ou igual ao número de ordem que o artigo ocupa (Grupo Scimago, 2006).

O índice apresenta algumas características: é um valor que nunca decresce no decorrer da trajetória de um pesquisador, porém, à medida que se avança no valor do índice, requer dele um maior esforço; seu aumento não é linear, pois o indicador não é totalmente influenciado pelo número de trabalhos publicados, mas está fortemente associado ao número de citações; seu valor depende da natureza da área do pesquisador. Pesquisadores advindos de áreas que não são mainstream não alcançarão valores altos de índice $\mathrm{h}$, como aqueles que trabalham em áreas mais destacadas da ciência.

Além disso, estudos anteriores comprovam que trabalhos em coautoria aumentam a produtividade e o impacto dos pesquisadores para a área. Esses trabalhos são mais presentes nas áreas mainstream, onde se encontram os maiores índices $\mathrm{h}$.

Segundo Hirsch (2005, p. 4), "a high $\mathrm{h}$ is a reliable indicator of high accomplishment, the converse is not necessarily always true".

Hirsch defende que o índice $\mathrm{h}$ é preferível aos outros indicadores anteriormente citados, também de valor pontual - número total de artigos, número total de citações e média de citações por artigo -, classicamente usados para avaliar a produção científica de um pesquisador, por se constituir em um número simples e fácil de calcular, por medir o impacto e a importância dos artigos, e por ser uma estimativa robusta da contribuição global do pesquisador para a área, na medida em que não é influenciado por valores extremos, isto é, pelo conjunto de artigos não citados, pouco citados ou altamente citados.

Os estudiosos têm sido unânimes em considerar o índice h uma medida que tende a valorar o esforço científico, ao levar em conta todo o percurso acadêmico do pesquisador, de forma cumulativa.

No entanto, Hirsch destaca que um número único valor nunca será suficiente para descrever 
amplamente o perfil multifacetado de um pesquisador, sendo necessária a combinação de outros indicadores na avaliação do mesmo.

Rousseau (2008, p. 2) apresenta vantagens e desvantagens referentes ao índice $h$. Entre as primeiras, destaca, entre outras, que o índice $h$ encoraja trabalhos de alta qualidade, combina dois indicadores: índice de produtividade, pelo número de publicações, e visibilidade, pelo número de citações. Entre as desvantagens, considera que o número de coautores pode influenciar o número de citações, potencializando o índice $\mathrm{h}$, além de que este pode ser influenciado pela auto-citação. O autor apresenta ainda em relação à natureza do próprio índice que o mesmo discrimina somente os melhores cientistas da área e que, em virtude de acumular o número de citações, o índice $\mathrm{h}$ de determinado pesquisador pode aumentar ainda que o mesmo nada mais produza.

Outros pesquisadores apresentam estudos relativos às propriedades matemáticas do índice $h$ (Glänzel (2006) e a extensão do uso deste índice não somente aos pesquisadores individualmente, mas também a periódicos (Braun, Glänzel e Schubert, 2006), por países (Csajbók, Berhidi, Vasas e Schubert, 2007) e por área (Iglesias e Pecharromán, 2007).

Considerando as questões arroladas, pesquisadores têm aplicado o índice $\mathrm{h}$ nas diferentes áreas do conhecimento, comparando-o com os indicadores clássicos. Destacam-se Grupo Scimago (2007), Cronin e Meho (2006), Van Raan (2006), Kulasegarah e Fenton (2009) e Mugnaini, Packer, e Meneghini (2008).

\section{Metodologia}

A partir do trabalho realizado por Grácio, Oliveira e Matos (2009), foram selecionados os autores mais citados na temática "Estudos Métricos", nos periódicos da área de Ciência da Informação da biblioteca eletrônica SciELO.

Dos pesquisadores citados nos 53 artigos analisados que trataram especialmente do tema em questão, foram destacados 35 autores considerados mais citados pelas autoras por terem pelo menos 6 citações.

Trabalhou-se com esse universo, considerando que, sob a perspectiva dos pesquisadores brasileiros da Ciência da Informação, os 35 autores constituem a frente de pesquisa na temática em questão.
Para cada um deles, buscaram-se, em fevereiro de 2010, na base de dados Web of Science, o índice h, o total de artigos publicados, o número total de citações, a média de citações e o número de citações do artigo mais citado.

Delimitou-se a produção de cada autor, segundo os seguintes parâmetros:

- na categoria "tipo de documentos", somente os artigos publicados;

- na seleção temporal, usou-se a opção "all years";

- controle de homonímia: utilização da ferramenta de seleção de autor disponível na base de dados.

Neste último caso, ainda havendo dúvidas sobre os artigos produzidos (se são de pesquisadores homônimos), buscou-se, na lista da produção arrolada, um artigo que, pelo periódico e temática, fosse reconhecidamente do autor em questão. Refinou-se novamente a seleção, acessando o nome do autor naquela publicação. Por este mecanismo, a Web of Science retorna somente à lista de produção do autor específico. Além disso, quando necessário, devido a nome de pesquisadores comuns em determinada língua, incluiu-se, na busca, o refinamento "adress". Procedeu-se dessa forma para todos os pesquisadores brasileiros.

Neste levantamento, do total de 35 autores mais citados, 3 pesquisadores brasileiros não foram encontrados na base de dados em questão, trabalhando-se com um total de 32 pesquisadores.

Dada a natureza cumulativa do índice $\mathrm{h}$, no levantamento na Web of Science levou-se em consideração toda a produção científica do pesquisador, independente da área.

Os dados levantados foram apresentados em tabela do software Excel. Calculou-se a correlação de Pearson entre os indicadores, índice $h$, total de artigos publicados, número total de citações, média de citações e número de citações do artigo mais citado, também por meio do software Excel.

\section{Apresentação e análise dos dados}

A análise da Tabela I mostra uma amplitude bastante grande de variação para todos os indicadores sobre análise: o índice $\mathrm{h}$ varia de 0 até 44; o total de artigos publicados, de 1 até 805; o número total de citações, de 0 até 9.489 ; a média de citações, entre 0 e 97,8; e o número de citações do artigo mais citado é 789 . 


\begin{tabular}{|c|c|c|c|c|c|}
\hline Pesquisadores (País) & Índice $\mathrm{h}$ & Total de artigos & Total de citações & Média de citações & Artigo mais citado \\
\hline Newman, M. E. J. (EUA) & 44 & 97 & 9489 & 97,8 & 789 \\
\hline Garfield, E. (EUA) & 29 & 805 & 4586 & 5,7 & 789 \\
\hline Glänzel, W. (Bélgica) & 25 & 77 & 1522 & 19,8 & 153 \\
\hline Meneghini, R. (Brasil) & 20 & 44 & 1595 & 36,3 & 206 \\
\hline Thelwall, M. (Reino Unido) & 20 & 117 & 1290 & 11 & 86 \\
\hline Moed, H. F. (Holanda) & 19 & 42 & 1071 & 25,5 & 132 \\
\hline Rousseau, R. (Bélgica) & 18 & 106 & 1192 & 11,3 & 89 \\
\hline Cronin, B. (Eua) & 16 & 77 & 871 & 11,3 & 74 \\
\hline Ingwersen, P. (Dinamarca) & 14 & 30 & 1078 & 35,9 & 280 \\
\hline Persson, O. (Suécia) & 11 & 18 & 492 & 27,3 & 111 \\
\hline Courtial, J.P. (França) & 11 & 29 & 463 & 16 & 115 \\
\hline Vinkler, P. (Hungria) & 11 & 20 & 321 & 16,1 & 58 \\
\hline Price, D. J. De S. (Eua) & 9 & 15 & 1444 & 96,3 & 651 \\
\hline Martin, B.R. (Reino Unido) & 7 & 15 & 440 & 29,3 & 191 \\
\hline Tague-Sutcliffe,J. (Canadá) & 7 & 21 & 173 & 8,2 & 35 \\
\hline Katz, J. S. (Reino Unido) & 6 & 7 & 347 & 49,6 & 191 \\
\hline Meadows, A. J. (Reino Unido) & 6 & 14 & 112 & 8 & 36 \\
\hline Herrero-Solana, V. (Espanha) & 6 & 16 & 85 & 5,3 & 31 \\
\hline Jiménez-Contreras, E. (Espanha) & 5 & 17 & 83 & 4,9 & 23 \\
\hline Wormell, I. (Suécia) & 4 & 17 & 78 & 4,6 & 38 \\
\hline Moya-Anegón,F (Espanha) & 4 & 19 & 67 & 3,5 & 31 \\
\hline Velho, L. (Brasil) & 3 & 12 & 61 & 4 & 27 \\
\hline Macias-Chapula, C. A. (México) & 3 & 4 & 26 & 6,5 & 13 \\
\hline Braga, G. M. (Brasil) & 3 & 4 & 23 & 5,8 & 10 \\
\hline Quoniam, L. (França) & 2 & 10 & 11 & 1,1 & 3 \\
\hline Robredo, J. (Brasil) & 1 & 3 & 10 & 3,3 & 9 \\
\hline Spinak, E. (Uruguai) & 1 & 2 & 8 & 4 & 8 \\
\hline Rostaing, H. (França) & 1 & 1 & 3 & 3 & 3 \\
\hline Mueller, S. P.M. (Brasil) & 0 & 1 & 0 & 0 & 0 \\
\hline Noronha, D. P. (Brasil) & 0 & 2 & 0 & 0 & 0 \\
\hline Pinheiro, L.V.R. (Brasil) & 0 & 2 & 0 & 0 & 0 \\
\hline Cunha, M. B. Da (Brasil) & 0 & 1 & 0 & 0 & 0 \\
\hline
\end{tabular}

Tabela I. Indicadores dos pesquisadores presentes na Web of Science.

Observa-se que $38 \%$ dos pesquisadores têm índice h maior que 10, destacando-se: Newman (EUA), com $h=44$, que atua principalmente em análise de redes sociais; Garfield (EUA), com h = 29, um dos pioneiros de análise de citações; Glanzel (Bélgica), com $h=25$, coeditor do Scientometrics, um dos mais relevantes periódicos da área em estudo, atua com trabalhos de cunho analítico, utilizando recursos matemáticos; Thelwall (Reino Unido) e Meneghini (Brasil), com $\mathrm{h}=20$, este último o brasileiro com o maior índice $\mathrm{h}$ no conjunto estudado. Segundo classificação de Hirsch (2005), Newman é considera- do, pelo seu alto índice h, um pesquisador proeminente, e os demais são os bem sucedidos.

Ainda em relação ao índice $h$, quatro pesquisadores brasileiros apresentam índice zero. Três deles - Muller, Noronha e Pinheiro - têm publicação somente a partir de 2007 na Web of Science, o que sugere pouco tempo para um maior reconhecimento internacional.

$\mathrm{Na}$ Tabela I, os pesquisadores foram organizados em ordem decrescente do índice $h$, por ser este o foco desta pesquisa. Entretanto, se outros indicadores fossem também levados em 
consideração e colocados na mesma ordem, a organização dos pesquisadores seria outra. Assim, por exemplo, se considerada a média de citações, os pesquisadores em destaque seriam Newman, Price, Katz Meneghini e Ingwersen: os dois primeiros, com média em torno de 97 citações por artigo; o terceiro, com média de 50 citações por artigo; e os dois últimos, com média de, aproximadamente, 36 citações por artigo. Entretanto, para todos os indicadores em questão, exceto o total de artigos publicados (que indica produção), Newman é o pesquisador de maior destaque.

Apresenta-se a Tabela II com os resultados das correlações entre os cinco indicadores.

\begin{tabular}{lrrrrr}
\hline \multicolumn{1}{c}{ Indicadores } & $\begin{array}{c}\text { Índice } \\
h\end{array}$ & $\begin{array}{c}\text { Tot. } \\
\text { artigos }\end{array}$ & $\begin{array}{c}\text { Tot. } \\
\text { citações }\end{array}$ & $\begin{array}{c}\text { Média } \\
\text { cit. }\end{array}$ & $\begin{array}{l}N^{\circ} \\
\text { cit. } \\
\text { art. }\end{array}$ \\
\hline Índice $h$ & 1 & & & & \\
\hline Tot. artigos & 0,52 & 1 & & & \\
\hline Tot. citações & 0,87 & 0,49 & 1 & & \\
\hline Média cit. & 0,56 & $-0,01$ & 0,64 & 1 & \\
\hline$N^{\circ}$ cit. art. & 0,74 & 0,62 & 0,84 & 0,75 & 1 \\
\hline
\end{tabular}

Legenda: Tot. artigos = total de artigos publicados pelo pesquisador; Tot. citações $=$ número total de citações recebidas pelo pesquisador; Média cit. $=$ média de citações por artigo do pesquisador; $\mathrm{N}^{\circ}$ cit. art. = número de citações do artigo mais citado.

Tabela II. Matriz simétrica com os valores da correlação de Pearson ( $r$ )

Com base nos resultados da Tabela II, nota-se moderada correlação entre 0 índice $\mathrm{h}$ e o total de artigos publicados. $O$ valor de $r=0,52$ indica que não há relação significativa entre estes dois indicadores, o que sugere que a dependência entre estas variáveis é frágil, uma vez que um alto índice $\mathrm{h}$ pressupõe alta produtividade, entretanto a recíproca não é verdadeira. O mesmo ocorre entre $\mathrm{o}$ índice $\mathrm{h}$ e a média de citações, com $r=0,56$.

Por outro lado, destaca-se a alta correlação entre índice $\mathrm{h}$ e o total de citações, com $\mathrm{r}=$ 0,87 , significando uma forte dependência entre as duas variáveis.

Há ainda uma correlação frágil entre o total de artigos publicados e o número total de citações, com $r=0,49$, indicando que alta produtividade não implica necessariamente em alto impacto $e$ inserção do pesquisador.

Resultados similares às quatro correlações apontadas nos parágrafos anteriores foram encontrados por Grupo Scimago (2007).

Destaque-se ainda a correlação significativa entre o índice h e o número de citações do arti- go mais citado $(r=0,74)$. Apesar de o índice $h$ ser considerado um indicador robusto e resistente a valores extremos, entre eles os chamados "big hits", entende-se que o fato de um autor ter um artigo com alta frequência de citação possivelmente exerça influência no número de citações das demais produções, o que por sua vez eleva o valor do índice $\mathrm{h}$. Constata-se aqui, novamente, o efeito Mateus, padrão de comportamento observado em vários fenômenos da Ciência da Informação, desde seus primórdios.

Em relação à correlação entre o número de citações do artigo mais citado e os outros indicadores, destaque-se a correlação entre o artigo mais citado e a quantidade de citações $(r=$ 0,84 ), bem como entre o artigo mais citado e a média de citações $(r=0,75)$. Estas associações são compreensíveis, dada a própria natureza das variáveis correlacionadas, que são intrinsecamente ligadas. Particularmente, a correlação entre o artigo mais citado e a média decai em relação à primeira, na medida em que a média é influenciada pelos artigos sem citação.

Cabe ressaltar a ausência de correlação entre o total de artigos publicados e média de citação por artigo $(r=-0,01)$, o que indica a independência entre estes indicadores.

Registrou-se também um pesquisador que apresentou produção total bem superior e distinta dos demais na base Web of Science. Considerando o destaque deste pesquisador em relação a variável quantidade de publicações, levantouse a produção do mesmo, tanto no "All Database" do próprio Thomson Reuters-ISI como em outra base, obtendo-se nestas duas últimas bases quantidade de produção próxima e bem abaixo da encontrada na Web of Science. Porém, nas três bases, o índice $\mathrm{h}$ se manteve bem próximo, uma vez que as publicações arroladas na Web of Science, e não presente nas demais, não apresentaram citações. Em consequência disso, algumas correlações entre os indicadores analisados podem estar subestimadas.

\section{Considerações finais e recomendações}

Em vista dos dados obtidos na Tabela I e Tabela II, matriz de correlação, considera-se que o índice $\mathrm{h}$ apresenta correlação minimamente moderada com todos os outros indicadores, uma vez que a correlação variou de 0,52 até 0,87 , o que indica uma inter-relação entre as ideias subjacentes a estes indicadores.

Em síntese, as correlações apresentadas entre os indicadores (algumas mais frágeis, outras mais significativas) mostram que entre as variá- 
veis estudadas existe, em alguns casos, maior dependência entre elas e em outros tantos, mais fragilidade de relação, isto é, menor dependência entre as mesmas. Nesta última situação, entende-se que o índice $\mathrm{h}$, isolado ou separadamente, representa parcialmente o perfil do pesquisador.

Isoladamente, o índice h é o indicador que mais se aproxima de uma descrição do perfil de um pesquisador ao longo de sua vida. Entretanto, para se retratar de forma plena a produção de um pesquisador, os outros indicadores combinados dão um retrato mais verdadeiro do mesmo, oferecendo informações complementares ao índice $\mathrm{h}$.

Cumpre lembrar que o índice $\mathrm{h}$ não é um valor sempre único e absoluto, pode diferir de acordo com a base de dados consultada. Entretanto as discrepâncias mostram-se pequenas e os valores do índice $\mathrm{h}$ ficam sempre próximos um do outro.

Esta pesquisa mostrou a baixa presença de periódicos publicados em línguas latinas na lista das bases do Thomson Reuters-ISI.

Finalizando, recomenda-se que se dê continuidade a este estudo, propondo-se a análise do índice h com outras variáveis, tais como "ano da primeira publicação" e "ano da primeira citação" e com medidas de variabilidade da publicação de cada autor. Sugere-se também que a pesquisa em questão seja realizada em outras bases de dados e se estenda a outros temas.

\section{Referências}

Base SciELO (1998). Scientific Electronic Library. http://www.scielo.br. ( 2009-03-30).

Braum, Tibor; Glänzel, Wolfgang; Schubert, András (2006). A Hirsch-tipe index for journals. // Scientometrics. 69:1 (2006) 169-173.

Cronin, Blaise; Meho, Lokman (2006). Using the h-index to rank influential information scientists. // Journal of the American Society for Information Science and Technology. 57:9 (may 2006) 1275-1278.

Csajbók, Edit; Berhidi, Anna; Vasas, Lívia; Schubert, András (2007). Hirsch-index for countries based on Essential Science Indicators data. // Scientometrics. 73:1 (2007) 91-117.
Dalpian Juliana; Noronha, Daisy Pires (2009). Índice de Hirsch: apresentação e aplicabilidade no campo da Ciência da Informação. // Anais do X Encontro Nacional de Pesquisa da ANCIB-ENANCIB, João Pessoa, Br, 2009. João Pessoa: ANCIB, 2009. 2136-2150.

Glänzel, Wolfgang (2006). On the h-index - A mathematical approach to a new measure of publication activity and citation impact. // Scientometrics. 67:2 (2006) 315-321.

Grácio, Maria Cláudia Cabrini; Oliveira, Ely Francina Tannuri de; Matos, Gislaine I. de (2009). Visiblidade dos pesquisadores no tema Estudos Métricos: análise de citação e co-citação nos periódicos do SciELO. // Ibersid. (2009) 81-86.

Grupo Scimago (2006). El índice h de Hirsch: aportaciones a un debate. // El profesional de la información. 16:1 (enero-febrero 2007) 47-49.

Grupo Scimago (2007). El índice h de Hirsch: su aplicación a algunos de los científicos españoles más destacados. // El profesional de la información. 15:4 (julio-agosto 2006) 304-306.

Hirsch, Jorge E. (2005). An index to quantify an individual's scientific research output. // ArXiv:physics/0508025v5 [physics.soc-ph]. (September 2005) http://arxiv.org/ abs/physics/0508025 (2009-04-18).

Inglesias, Juan E.; Pecharromán, Carlos (2007). Scaling the h-index for different scientific ISI fields. // Scientometrics. 73:3 (2007) 303-320.

Kulasegarah, Jeyanthi; Fenton, John E. (2009). Comparison of the $\mathrm{h}$ index with standard bibliometric indicators to rank influential otolaryngologists in Europe and North America. // European Archives of Otorhinolaryngology. 267 (June 2009) 455-458.

Meneghini, Rogério (1998). Avaliação da produção científica e o projeto SciELO. // Ciência da Informação. 27:2 (maio-ago. 1998) 219-220.

Mugnaini, Rogério; Packer, Abel Laerte; Meneghini, Rogério (2008). Comparison of scientists of the Brazilian Academy of Sciences and of the National Academy of Sciences of the USA on the basis of the h-index. // Brazilian Journal of Medical and Biological Research. 41:4 (abril 2008) 258-262. http://www.scielo.br/pdf/bjmbr/v41n4 16971.pdf (2010-02-15).

Rousseau, Ronald (2008). Reflections on recent developments of the h-index and h-type indices. // Collnet Journal of Scientometrics and Information Management. 2:1 (June 2008) 1-8.

Van Raan, Anthony F. J. (2006). Comparison of the Hirschindex with standard bibliometric indicators and with peer judgment for 147 chemistry research groups. // Scientometrics. 67:3 (2006) 491-502.

Recibido: 18-06-2010.

Aceptado: 18-06-2010. 
